\title{
Ethnographic research applied to anesthesia
}

Jan Angus PhD

$\mathrm{E}$ THNOGRAPHIC inquiry has a history in health care research that spans several decades, yet discussions of its fundamental principles have only recently emerged in the mainstream medical literature. ${ }^{1,2}$ Since evidence for best practices is derived from both quantitative and qualitative sources, ${ }^{3}$ it is worthwhile to explore some of the intellectual and methodological foundations of ethnographic studies like Smith et al.'s paper in this issue, "Communication between anesthesiologists, patients and the anesthesia team: a descriptive study of induction and emergence". ${ }^{4}$ I will begin with a discussion of the foundations of ethnographic research, then will highlight specific indicators of quality found in their paper. This introduction is intended to draw attention to a growing body of ethnographies that illuminate expert practice in anesthesia.

Ethnography is not a distinct "method", nor is it a system of procedures for data collection and analysis. Instead, it is a general approach to the study of social settings and practices that has its roots in several disciplines, especially anthropology, sociology and education. ${ }^{5,6}$ Some ethnographic studies in the health sciences may retain an anthropological focus on the health beliefs and behaviours of specific ethnic groups. However, the focus has increasingly turned to health related beliefs and activities within social subcultures or interest groups - such as specialized health professions - that are distinguished more by their common goals or experiences than by shared ethnic origins. ${ }^{2,7}$ Hence, the complexities of anesthetic practice have inspired several recent ethnographies. ${ }^{8-10}$

Ethnographers are particularly interested in the various forms of knowledge that shape people's activities within specific situations, recognizing that the deep inculcation of social competencies and skills through processes of socialization may imbue stores of tacit or habitual knowledge. ${ }^{10}$ In ethnographies of health care, this interest manifests itself in study of the contrast between the explicit or codified knowledge that underpins formal education in the health sciences and the tacit knowledge embedded in expert practice of health professionals. ${ }^{9-11}$ Inquiry is grounded in the assumption that people act in rational ways, but their systems of rationality or logic are inextricably contextdependent or situated. 7 One goal of ethnographic fieldwork is to understand and describe how logics and practices make sense within their particular social contexts. The pre-reflexive or tacit nature of the insider's knowledge of social situations poses a challenge to this aim; people are often remarkably inarticulate when asked to explain or describe specific reasons for some everyday decisions or activities because the underlying logics are part of an assumptive order that facilitates team work and rapid decision making. ${ }^{10}$

Ethnographic inquiry may rely on diverse techniques of data collection, but three of the most prominent methods are: extended periods of observation, formal or informal interviews and examination of written texts or other cultural artifacts. ${ }^{6,12}$ A hallmark of ethnographic inquiry is the selective combination of two or more sources of data to develop detailed descriptions and interpretations of activities or events. Analysis of the data is concurrent with its collection and, as understanding of the phenomenon evolves, it is common for the design of an ethnographic study to shift in response. New questions may be added to interviews to help clarify researchers' interpretations of their observations, or additional related situations may be observed to learn if similar patterns are present. Sampling is therefore not guided by notions of generalizability, but by a search for the most appropriate sources of information to answer the investigators' questions. In this sense, ethnographic sampling strategies are similar to purposive sampling, but they reflect an iterative engagement between fieldwork and the evolving explanatory theory that is the product of the inquiry.

In their paper, Smith et al. ${ }^{4}$ argue that the ethnographic approach is ideal for illuminating the tacit

From the Faculty of Nursing, University of Toronto, Toronto, Ontario, Canada.

Address correspondence to: Dr. Jan Angus, Faculty of Nursing, University of Toronto, 50 St. George Street, Toronto, Ontario M5S 3H4, Canada. Phone: 416-978-0695; Fax: 416-978-8222; E-mail: an.angus@utoronto.ca 
knowledge developed by professionals in the practice world, and their study presents some intriguing insights into communication during induction and emergence. The authors have taken an original approach to the issue of communication by seeking to reveal patterns of communication in everyday practice. Rather than simply interviewing anesthesiologists, the authors have observed communication during induction and emergence, thus accommodating the pre-reflexive nature of interpersonal skill. This makes the study somewhat of a "gem" since many qualitative health researchers are concerned about the relative paucity of observational studies that deal with professional practice, noting that interviews alone may not be sufficient. People do not always describe in interviews everything that they actually do, not because they are disingenuous but because, as explained above, they do not always reflect fully on the minutiae of everyday activity. Tacit knowledge, by its very nature, often cannot be communicated. ${ }^{13}$

Further, there is considerable evidence that the study was conducted in a systematic and rigorous manner. Several authors have discussed the issue of quality in ethnographic research and identify specific indicators. ${ }^{14,15}$ Prolonged engagement with the field is one such indicator; there must be sufficient opportunities to observe the phenomenon of interest to ensure a detailed description is obtained. Although study participants may initially alter their behaviour in response to the presence of an observer, prolonged engagement increases the likelihood that they will revert to usual routines. The authors observed participating anesthesiologists approximately three times per month over the course of one year for a total of $133 \mathrm{hr}$ - enough time to develop familiarity with these respondents' practices. In addition, team members were interviewed about patterns seen during field observations. Another indicator of quality in ethnographic research is evidence of systematic documentation of observations as fieldnotes; in fieldwork the authors follow the traditional pattern of making initial brief notations while observing, then expanding the notes immediately afterward. Some investigators will also develop observation guides to further hone their attention to particular facets of the situation, ${ }^{16}$ however in this case, the overall focus on tacit knowledge in expert practice is all encompassing, and an observation guide might arguably constrain discovery.

The analysis is mainly grounded in field observations and field notes, but also draws on pertinent statements from ensuing interviews with respondents. Corroborative quotes and excerpts are included in the paper to allow readers the opportunity to exam- ine examples of the evidence supporting the authors' conclusions. This is an important feature of all qualitative reports, and in this case we are provided with several exemplars. Yet it is noteworthy that the limits on manuscript length and format that prevail among mainstream medical journals places ethnographers in a quandary; they must choose between supplying ample corroborative evidence or providing sufficient interpretive discussion of this evidence to allow readers to follow the analysis. Overall, the authors strike a balance between these conflicting imperatives, but I found myself wishing for elaboration of some points. For example, the intriguing presence of metaphors in communication with patients during induction and the use with adult patients of terms and images commonly reserved for conversation with children implies evocation of very specific experiences and relationships for a particular purpose. Might references to childhood bedtime stories be intended to relax patients with images of a benign parental influence, for example? If so, there is one perspective missing from this analysis - that of the patients who underwent induction. No one ethnographic study can supply all views of the phenomenon simultaneously; further study is warranted to bring patient experiences forward.

To further strengthen analysis of ethnographic field observations, a participatory approach is frequently used; study respondents may be invited to assist with data analysis or to verify and expand on findings. Use of this strategy in the present study is an indication that the investigators took steps to fully explore insiders' experiences and understandings of expert anesthetic practice. Further description of how participants were involved and the nature of their contributions to the analysis would be helpful here, although the authors refer to this process in greater detail elsewhere. ${ }^{8,9}$

The authors attended to negative cases or "discordant data" in their analysis, another indicator of a rigorous approach to ethnographic inquiry. As ethnographers begin to find semi regular patterns within their observations, they search for examples that contradict these patterns in order to understand more fully the underlying mechanisms. In the section entitled "Appropriate and inappropriate communication", examples are provided to demonstrate how participants' responses to disruptions to the assumptive routines of communication enabled the authors to develop a deeper grasp of the multiple functions of seemingly mundane verbalizations. These examples show how the entire operative team may orient their activities around the signals provided by an anesthesiologist's remarks to the patient during induction; when these signals are absent, other team members 
reported discomfort because they were not prepared to smoothly coordinate their own actions with those of the anesthesiologist. The authors' inclusion of this section is commendable, since this degree of analytic detail is often lacking in qualitative literature.

In summary, ethnographic research employs methods different from those more typically encountered in the pages of this journal, but it facilitates access to evidence that is beyond the scope of other approaches. This introduction to ethnography has included a brief discussion of its foundational tenets and methodological features; it has also highlighted indicators of quality in ethnographic research using Smith et al. "Communication between anesthesiologists, patients and the anesthesia team: a descriptive study of induction and emergence" as an illustrative example. As the program of research pursued by these authors demonstrates, much can be learned from ethnographic study of expert practice in anesthesia.

\section{La recherche ethno- graphique appliquée à l'anesthésie}

L'enquête ethnographique fait partie de la recherche en soins de santé depuis des décennies, mais la réflexion sur ses principes fondamentaux n'a que récemment émergé du flot des publications médicales. ${ }^{1,2}$ La preuve des meilleurs pratiques étant dérivée de sources à la fois quantitatives et qualitatives, ${ }^{3}$ il est intéressant d'explorer certains principes intellectuels et méthodologiques des études ethnographiques comme le font Smith et coll. dans leur article du présent numéro, Communication between anesthesiologists, patients and the anesthesia team: a descriptive study of induction and emergence. ${ }^{4}$ Nous discuterons d'abord des fondements de la recherche ethnographique et présenterons des indicateurs spécifiques de la qualité découverts dans l'article. Cette introduction à l'approche ethnographique veut attirer l'attention sur une composante de l'ethnographie en pleine évolution qui éclaire la pratique spécialisée de l'anesthésie.

L'ethnographie n'est pas une «méthode» distincte, ni un système de procédures pour la cueillette et l'analyse de données. C'est plutôt une approche générale pour l'étude de situations et pratiques sociales qui ont leurs racines dans diverses disciplines, dont l'anthropologie, la sociologie et l'éducation..$^{5,6}$ Dans certaines études ethnographiques en sciences de la santé, on peut adopter un point de vue anthropologique sur les croyances entourant la santé et les comportements de groupes ethniques spécifiques. Mais, de plus en plus, l'attention est centrée sur les croyances et les activités de sous-cultures sociales ou de groupes d'intérêt - comme les professions spécialisées en santé - qui se distinguent plus par leurs expériences ou objectifs communs que par leur origine ethnique commune. ${ }^{2,7}$ En conséquence, les complexités de la pratique anesthésique ont inspiré plusieurs études ethnographiques récentes. ${ }^{8-10}$

Les ethnographes sont particulièrement intéressés aux diverses formes de connaissances qui façonnent les activités des gens dans des situations spécifiques, reconnaissant que l'inculcation profonde de compétences et d'habiletés sociales par des procédés de socialisation peut animer le contenu des connaissances tacites ou habituelles. ${ }^{10}$ Dans les études ethnographiques en santé, cet intérêt se manifeste dans l'étude du contraste entre le savoir explicite ou codifié qui soutient l'éducation formelle en sciences de la santé et le savoir tacite intégré dans la pratique spécialisée des professionnels de la santé. 9-11 $^{-1}$ L'enquête est fondée sur l'idée que les gens agissent de façon rationnelle, mais que leur système de rationalité ou de logique dépend absolument du contexte, ou s'y situe. ${ }^{7}$ L'un des buts du travail ethnographique est de comprendre et de décrire comment la logique et la pratique ont du sens dans leurs contextes sociaux particuliers. La nature préréflexive, ou tacite, des connaissances de l'expert sur les situations sociales pose un défi à l'atteinte de ce but; les gens sont souvent très confus quand on leur demande d'expliquer ou de décrire les raisons spécifiques des décisions prises ou des activités réalisées quotidiennement parce que la logique qui les sous-tend fait partie d'une commande présumée qui facilite le travail d'équipe et la prise de décision rapide. ${ }^{10}$

L'enquête ethnographique peut reposer sur diverses techniques de collection de données, mais les trois plus importantes sont de longues périodes d'observation, des entrevues formelles et informelles et l'examen de textes écrits ou d'autres objets culturels. ${ }^{6,12}$ Une caractéristique de l'enquête ethnographique est la combinaison sélective de deux sources de données ou plus pour développer des descriptions détaillées et des interprétations des activités ou des événements. L'analyse des données est concurrente à leur cueillette et à mesure que la compréhension du phénomène évolue, il arrive souvent que la conception de l'étude ethnographique se transforme en conséquence. De 
nouvelles questions peuvent s'ajouter aux entrevues pour aider à clarifier les interprétations que les chercheurs font de leurs observations, ou des situations supplémentaires apparentées peuvent être observées pour savoir si des modèles similaires sont présents. L'échantillonnage n'est plus guidé alors par des notions de généralisabilité, mais par une recherche des sources les plus appropriées d'informations pour répondre aux questions des chercheurs. En ce sens, les stratégies d'échantillonnage ethnographique sont semblables à l'échantillonnage dirigé, mais elles traduisent un engagement répété entre le travail sur le terrain et la théorie explicative en évolution qui est le produit de l'enquête.

Dans leur article, Smith et coll. ${ }^{4}$ soutiennent que l'approche ethnographique est idéale pour mettre en lumière le savoir tacite développé par les professionnels dans le monde de la pratique. Leur étude présente certains aspects curieux de la communication qui a lieu pendant l'induction de l'anesthésie et le retour à la conscience. Les auteurs ont abordé la question de la communication d'une façon originale en cherchant à découvrir quels modèles ont cours dans la pratique quotidienne. Plutôt que d'interroger simplement les anesthésiologistes, les auteurs ont observé la communication pendant l'induction et le retour à la conscience, intégrant ainsi la nature préréflexive des compétences interpersonnelles. Cette approche rend l'étude précieuse, car de nombreux chercheurs de qualité dans le domaine de la santé sont préoccupés par l'absence relative d'études par observation concernant la pratique professionnelle et ils remarquent que les entrevues seules ne suffisent pas. Au cours d'une entrevue, les gens ne décrivent pas toujours tout ce qu'ils font vraiment, non parce qu'ils sont malhonnêtes mais parce qu'ils ne rendent pas toujours compte exactement de la minutie de l'activité quotidienne. La connaissance tacite, de par sa nature propre, souvent ne peut être communiquée. ${ }^{13}$

De plus, il y a une preuve impressionnante de la rigueur et de la systématisation de l'étude. Quelques auteurs ont examiné la question de la qualité en recherche ethnographique et désigné des indicateurs spécifiques. ${ }^{14,15}$ Un engagement prolongé sur le terrain est l'un de ces indicateurs; il doit y avoir suffisamment d'occasions d'observer le phénomène d'intérêt pour s'assurer qu'une description détaillée sera obtenue. Même si les participants peuvent modifier leur comportement au début de l'étude, en réaction à la présence d'un observateur, un engagement de longue durée accroît la probabilité d'un retour à la routine. Les auteurs ont observé les anesthésiologistes participants environ trois fois par mois pendant un an, pour un total de $133 \mathrm{~h}$. C'est un délai suffisant pour que la pratique des répondants devienne familière. De plus, les membres de l'équipe ont été interrogés sur les modèles utilisés pendant les observations de terrain. Un autre indicateur de qualité est la preuve de documentation systématique des observations sous forme de notes d'opérations; dans le travail sur le terrain, les auteurs suivent le modèle traditionnel de brèves notes initiales rédigées pendant l'observation, suivies de notes plus complètes immédiatement après. Certains chercheurs vont aussi élaborer des guides d'observation pour raffiner l'examen de facettes particulières de la situation, ${ }^{16}$ malgré que dans le cas présent l'optique générale sur la connaissance tacite de la pratique des professionnels recouvre tout et un guide d'observation risquerait de restreindre la découverte.

L'analyse est principalement fondée sur les observations de terrains et les notes d'opérations, mais est aussi élaborée à partir des déclarations pertinentes provenant d'entrevues ultérieures avec les répondants. Des citations et des extraits corroborants, inclus dans l'article, permettent au lecteur d'examiner des exemples de la preuve qui appuie les conclusions des auteurs. C'est un aspect important des articles qualitatifs et, dans le cas présent, nous bénéficions de plusieurs exemples. Cependant, les limites imposées par les principales revues médicales quant à la longueur et au format du manuscrit placent les ethnographes devant un dilemme ; ils doivent choisir entre fournir des preuves corroborantes abondantes ou une discussion interprétative suffisante de cette preuve pour permettre au lecteur de suivre l'analyse. Dans l'ensemble, les auteurs atteignent un équilibre entre ces impératifs contradictoires, mais nous désirons préciser certains points. Par exemple, la présence intrigante de métaphores dans la communication avec les patients pendant l'induction et l'usage avec les adultes de termes et images habituellement réservés à la conversation avec des enfants implique l'évocation d'expériences et de relations très spécifiques pour un objectif particulier. Les références aux histoires de chevet de l'enfance ontelles pour but de détendre les patients avec des images d'une influence parentale anodine, par exemple ? Si c'est le cas, une perspective est absente de l'analyse - celle des patients qui reçoivent l'induction. Aucune étude ethnographique ne peut présenter simultanément toutes les visions d'un phénomène ; il faudra étudier l'expérience du patient.

Pour renforcer davantage l'analyse des observations ethnographiques sur le terrain, une approche participative est souvent utilisée ; les répondants de l'étude peuvent être invités à contribuer à l'analyse 
des données ou à vérifier et à développer les résultats. L'utilisation de cette stratégie dans la présente étude est une indication que les chercheurs ont pris les mesures nécessaires pour explorer pleinement les expériences des gens en place et les connaissances d'une pratique anesthésique experte. Une description plus poussée sur l'implication des participants et la nature de leurs contributions à l'analyse aurait été utile ici, même si les auteurs rappellent ce processus avec de plus amples détails ailleurs. ${ }^{8,9}$

Les auteurs ont prêté attention aux cas négatifs ou aux «données discordantes» dans leur analyse, un autre indicateur d'une approche rigoureuse de l'enquête ethnographique. Quand les ethnographes commencent à découvrir des modèles semi-réguliers dans leurs observations, ils recherchent des exemples qui contredisent ces modèles pour mieux comprendre les mécanismes sous-jacents. Dans la section intitulée "Communication appropriée et inappropriée», des exemples sont fournis pour démontrer comment les réactions des participants aux ruptures des routines de communication présumées permettent aux auteurs de développer une saisie plus approfondie des multiples fonctions des verbalisations apparemment banales. Ces exemples montrent comment toute l'équipe du bloc opératoire peut orienter ses activités autour des signaux fournis par les remarques d'un anesthésiologiste envers un patient pendant l'induction ; quand ces signaux sont absents, les autres membres de l'équipe se disent mal à l'aise parce qu'ils n'ont pas été préparés à coordonner régulièrement leurs propres actions avec celles de l'anesthésiologiste. L'inclusion de cette section par les auteurs est à souligner, car ce degré de détail analytique est souvent absent dans les publications qualitatives.

En résumé, la recherche ethnographique emploie des méthodes différentes de celles qu'on voit d'ordinaire dans les pages du journal, mais elle facilite l'accès à la preuve qui va au-delà du domaine des autres approches. Cette introduction à l'ethnographie comportait une brève discussion de ses croyances générales et de ses caractéristiques méthodologiques; elle a aussi mis en lumière des indicateurs de qualité en recherche ethnographique en utilisant l'article de Smith et coll. ${ }^{4}$ comme exemple illustré. Comme le montre le programme de recherche suivi par ces auteurs, on peut apprendre beaucoup d'une étude ethnographique de la pratique professionnelle de l'anesthésie.

\section{References}

I Atkinson P, Pugsley L. Making sense of ethnography and medical education. Med Educ 2005; 39: 228-34.

2 Savage J. Ethnography and health care. BMJ 2000;
321: 1400-2.

3 Upshur RE. The status of qualitative research as evidence. In: Morse JM, Swanson JM, Kuzel AJ (Eds). The Nature of Qualitative Evidence. Thousand Oaks, CA: Sage; 2001: 5-26.

4 Smith AF, Pope C, Goodwin D, Mort M. Communication between anesthesiologists, patients and the anesthesia team: a descriptive study of induction and emergence. Can J Anesth 2005; 52: 915-20.

5 Atkinson P, Coffey A, Delamont S, Lofland J, Lofland L. Handbook of Ethnography. London: Sage; 2001.

6 Hammersley M, Atkinson P. Ethnography: Principles in Practice, 2nd ed. London: Routledge; 1995.

7 Bloor $M$. The ethnography of health and medicine. In: Atkinson P, Coffey A, Delamont S, Lofland J, Lofland L. (Eds). Handbook of Ethnography. London: Sage; 2001: 177-87.

8 Smith AF, Mort M, Goodwin D, Pope C. Making monitoring 'work': human-machine interaction and patient safety in anaesthesia. Anaesthesia 2003; 58: 1070-8.

9 Smith A, Goodwin D, Mort M, Pope C. Expertise in practice: an ethnographic study exploring acquisition and use of knowledge in anaesthesia. Br J Anaesth 2003; 91: 319-28.

10 Hindmarsh J, Pilnick A. The tacit order of teamwork: collaboration and embodied conduct in anesthesia. Sociological Quarterly 2002; 43: 139-64.

11 Gabbay J, le May A. Evidence based guidelines or collectively constructed "mindlines?" Ethnographic study of knowledge management in primary care. BMJ 2004; 329: 1013.

12 LeCompte M, Schensul J. Designing \& Conducting Ethnographic Research. Walnut Creek, CA: AltaMira Press; 1999.

13 Eraut $M$. Non-formal learning and tacit knowledge in professional work. Br J Educ Psychol 2000; 70: 11336.

14 Mays N, Pope C. Qualitative research in health care. Assessing quality in qualitative research. BMJ 2000; 320: 50-2.

15 Giacomini MK, Cook DJ. Users' guides to the medical literature: XXIII. Qualitative research in health care A. Are the results of the study valid? Evidence-Based Medicine Working Group. JAMA 2000; 284: 357-62.

16 Spradley J. Participant observation. Orlando, FL: Harcourt Brace Jovanovich College Publishers; 1980. 